\title{
Epidemiological characteristics of peripheral circulating tumor cells and their relationship with the morbid state in gastric polyps and non- metastatic gastric cancer
}

Jun He

Tongren Hospital Shanghai Jiaotong University School of Medicine

\section{Yingjie Xu}

Tongren Hospital Shanghai Jiaotong University School of Medicine

\section{Peng Sun}

Tongren Hospital Shanghai Jiaotong University School of Medicine

\section{Chen Qian}

Tongren Hospital Shanghai Jiaotong University School of Medicine Jun Yan

Shanghai Jiao Tong University Affiliated Sixth People' Hospital

Chengguang Yang ( $\nabla$ sh6zhq@163.com)

Tongren Hospital, Shanghai Jiao Tong university School of Medicine https://orcid.org/0000-00015656-2051

\section{Research}

Keywords: Circulating tumor cells, gastric polyps, gastric cancer, tumor markers, pathological stages

Posted Date: June 22nd, 2020

DOI: https://doi.org/10.21203/rs.3.rs-37177/v1

License: (c) (1) This work is licensed under a Creative Commons Attribution 4.0 International License. Read Full License 


\section{Abstract}

\section{Background}

Circulating tumor cells (CTCs) can be detected in peripheral blood of many malignant tumor cases, but low numbers of CTCs can be detected in peripheral blood of healthy individuals as well as patients with benign tumors. It is not known whether peripheral CTC counts differ between patients with benign gastric diseases and those with gastric cancer. It is still unknown whether peripheral CTC counts can act as a superior factor to assess the clinicopathological stage in gastric cancer.

\section{Methods}

Comparative analysis of preoperative peripheral CTC counts was completed in patients with gastric polyps and non-metastatic gastric cancer. Correlation analysis was carried out between peripheral CTC counts and histological differentiation types, histopathological types, depth of tumor infiltration, positive lymph node metastasis rate and tumor markers (CEA, CA19-9 and CA72-4).

Results

Patients with gastric cancer had higher CTC counts than those with gastric polyps $(3.05 \pm 0.48 / 3.2 \mathrm{ml}$ vs $0.69 \pm 0.17 / 3.2 \mathrm{ml}, P<0.05)$. Over $84.62 \%$ of the higher CTC group $(\leq 1 / 3.2 \mathrm{ml})$ were gastric polyp patients. A significant difference was observed between lower CTC counts group $(\leq 1 / 3.2 \mathrm{ml})$ and higher CTC counts group $(>1 / 3.2 \mathrm{ml})$ diagnosed with either gastric polyps or gastric cancer $\left(X^{2}=9.67, P<\right.$ 0.005). Significant difference was observed between moderate differentiation and poor differentiation in gastric cancer $\left(X^{2}=2.43, P<0.05\right)$. The tissue pathological types distributions showed no statistically difference between two CTC counts group ( $P>0.05$ ). Significant CTC counts difference was observed between mucous membrane invasive group and muscular layer invasive group in gastric cancer (3.00 \pm $0.33 / 3.2 \mathrm{ml}$ vs $1.4 \pm 0.4 / 3.2 \mathrm{ml}, P<0.05)$. No significant CTC counts difference was observed in different positive lymph node ratios groups in gastric cancer $(P>0.05)$. CTC counts could show higher positive ratio when compared with CEA, CA19-9 and CA72-4 respectively $\left(X^{2}=34.37, X^{2}=36.89, X^{2}=29.72, P<0.001\right)$ or jointly $\left(X^{2}=16.71, P<0.001\right)$ in gastric cancer.

\section{Conclusions}

Peripheral CTC counts were different between patients with gastric polyps and patients with gastric cancer. CTC counts could act as an independent factor to assess the clinicopathological stage in gastric cancer. CTC counts might be more sensitive than routine tumor markers to reflect the existence of early gastric cancer.

\section{Introduction}


Gastric cancer, although the incidence of it varies around the world, is still a very common cancer worldwide [1-3], consequently radical surgery is still the preferred therapy method for patients with resectable gastric tumors. Although the current integrated therapies for gastric cancer have made a significant progress, there are still postoperative recurrence cases, many patients with stage II and III gastric cancers still exhibit tumor recurrence within one year following integrated therapies [4], so early detection and treatment is in highly demand. Circulating tumor cells (CTCs) can be detected at the preoperation and post-operation in peripheral blood in gastric cancer patients, the presence of post-operative peripheral blood CTCs may indicate the potential for tumor recurrence and metastasis [5-7].

The presence of CTCs suggests that a tumor may be present in vivo, and preoperative detection of peripheral blood CTCs can be used to assess early stage epidemiological characteristics and predictive values of circulating tumor cells in these patients with tumor [8-10]. When combining the clinicopathologic staging of the tumor, surgeons can comprehensively assess the necessity of subsequent treatments, such as chemotherapy and radiation.

However, a low number of CTCs can also be detected in the peripheral blood of some healthy individuals as well as patients with benign tumors [11-13]. Therefore, further studies are required to determine whether peripheral CTCs are differentially expressed in benign gastric disease and non-metastatic gastric cancer, and whether detection of CTCs accurately reflects the clinicopathologic characteristics of the primary tumor in benign and malignant gastric diseases. To address these questions, we carried out the comparative analysis of peripheral CTC counts in patients with gastric polyps and gastric cancer.

\section{Patients And Methods}

A total of 13 patients ( 5 males, 8 females) with gastric polyps, and 58 patients (40 males, 18 females) with gastric carcinoma were retrospectively studied at the department of surgery of Shanghai Tongren Hospital (Shanghai, China) between December 2014 and August 2016. After received patient consent and ethics committee recognition, peripheral CTCs quantification was performed the day before operation. Patient general information and pathological tumor characteristics are listed in Table 1. All tumor samples were identified as malignant epithelial tumors using Cytokeratin (AE1/AE3), carcinoembryonic antigen, Cytokeratin 7, and Cytokeratin 20. For carcinomas, the degree of tissue differentiation was classified as high, moderate, or poor differentiation, and tissue pathology was classified as papillary adenocarcinoma, tubular adenocarcinoma, papillary tubular adenocarcinoma, signet-ring cell carcinoma, or mucous adenocarcinoma, patients with tumor cells invasive depth from $T_{0}$ to $T_{4 a}$, lymph node positive from $N_{0}$ to $N_{3 b}$ and no tumor cells distant metastases $\left(M_{0}\right)$ were taken into accounted and subsequently observed. For polyps, degree oftissue differentiation was classified as low-grade intraepithelial neoplasia or high-grade intraepithelial neoplasia, and tissue pathology was classified as adenomatous polyp and proliferative polyps. Pathological consultations were completed by three experienced pathology specialists. 
Table 1

Characteristics of patients with gastric polyps or carcinoma

\begin{tabular}{|c|c|c|c|}
\hline Variable & Gastric polyps & Gastric carcinoma & $P$ Value \\
\hline Patients, n & 13 & 58 & \\
\hline \multicolumn{4}{|l|}{ Sex, n } \\
\hline Male & 5 & 40 & \multirow[t]{2}{*}{$P<0.05$} \\
\hline Female & 8 & 18 & \\
\hline Ages, years & $61.38 \pm 1.53(49-69)$ & $66.33 \pm 1.28(47-85)$ & $P>0.05$ \\
\hline \multicolumn{2}{|l|}{ Pathologic staging(TNM) } & \multicolumn{2}{|l|}{$\mathrm{T}_{0-4 \mathrm{a}} \mathrm{N}_{0-3 \mathrm{~b}} \mathrm{M}_{0}$} \\
\hline CTC counts(n/3.2 ml) & $0.69 \pm 0.17(0-2)$ & $3.05 \pm 0.48(0-16)$ & $P<0.05$ \\
\hline Tissue differentiation, $\mathrm{n}$ & 13 & 58 & \\
\hline \multicolumn{2}{|l|}{ High differentiated } & \multicolumn{2}{|l|}{0} \\
\hline \multicolumn{2}{|l|}{ Moderately differentiated } & \multicolumn{2}{|l|}{$12(20.69 \%)$} \\
\hline \multicolumn{2}{|l|}{ Poorly differentiated } & \multicolumn{2}{|l|}{$46(79.31 \%)$} \\
\hline LGIEN & \multicolumn{3}{|l|}{$5(38.46 \%)$} \\
\hline HGIEN & \multicolumn{3}{|l|}{$2(15.38 \%)$} \\
\hline NNP & \multicolumn{3}{|l|}{$6(46.16 \%)$} \\
\hline Tissue pathology, n & 13 & \multicolumn{2}{|l|}{58} \\
\hline Papillary adenocarcinoma & & \multicolumn{2}{|l|}{$7(12.07 \%)$} \\
\hline Tubular adenocarcinoma & & \multicolumn{2}{|l|}{$21(36.21 \%)$} \\
\hline Papillary tubular adenocarcinoma & & \multicolumn{2}{|l|}{$3(5.17 \%)$} \\
\hline Signet-ring cell carcinoma & & \multicolumn{2}{|l|}{$9(15.52 \%)$} \\
\hline Mucous adenocarcinoma & & \multicolumn{2}{|l|}{$18(31.03 \%)$} \\
\hline Adenomatous Polyp & \multicolumn{3}{|l|}{$7(53.84 \%)$} \\
\hline Proliferative Polyps & \multicolumn{3}{|l|}{$6(46.16 \%)$} \\
\hline \multicolumn{4}{|c|}{$\begin{array}{l}\text { Values are expressed as the mean } \pm \text { standard error of the mean.HGIEN, High-grade intraepithelial } \\
\text { neoplasia; LGIEN, Low-grade intraepithelial neoplasia; NNP, Non-neoplastic proliferation. CTC, } \\
\text { Circulating tumor cell. }\end{array}$} \\
\hline
\end{tabular}

\section{Materials And Chemicals}


Cytokeratin 7 (clone line OV-TL 12/30), Cytokeratin 20 (clone line SD33, mouse anti-human cell keratin) monoclonal antibodies, Cytokeratin (clone line AE1/AE3), carcinoembryonic antigen (clone line COL-1, mouse anti-human)monoclonal antibody,CA19-9(clone line 121SLE, mouse anti-human) monoclonal antibodies, were obtained from Biocare Medical (Walnut Creek, CA, USA). Human Cancer Antigen 72 - 4 (CA-724)antibody (mouse anti-human)was obtained from Sigma-Aldrich (MerckKGaA, Darmstadt, Germany).Horseradish peroxidase (HRP)-conjugated second antibody (goat anti-mouse) was obtained from Jackson Immuno Research Inc. (West Grove, PA, USA).LCD45-AF594 fluorescent antibody was obtained from Santa Cruz Biotechnology (Santa Cruz, CA, USA). Nuclear staining (DAPI) antibody was obtained from Beyotime Biotechnology (Shanghai, China).

\section{Circulating Tumor Cells}

CTCs were enriched by negative separation as described in previous studies [13, 14]. CD45 antibody beads were used to bind white blood cells, and all CTCs were enriched in precipitation. A total of $9.6 \mathrm{ml}$ of peripheral blood was obtained peripherally from each patient, across three collections $(3.2 \mathrm{ml}$ per collection) at three time points(8am, $2 \mathrm{pm}$, and $8 \mathrm{pm})$, using an indwelling sheathe syringe needle on the day before the operation. CTC counts were measured for each blood sample using fluorescence in situ hybridization in triplicate and mean values were adopted. The following criteria were used to identify CTCs: nuclear signal greater or equal to the triploid, positive nuclear DAPI staining, and negative CD45 staining [13]. Staining of cells was observed and counted using a fluorescence microscope (Nikon Cl-S, Tokyo, Japan). Peripheral blood CTC counts were measured for each $3.2 \mathrm{ml}$ blood sample using fluorescence in situ hybridization.

\section{Pathological Specimens}

Tumor tissues, lymph node and polyp samples were immediately fixed in formalin for 24 hand paraffin embedded, tissue sections were prepared as described in our previous study [13]. Primary antibodies (mouse anti human, 1:200) were added and incubated at $4{ }^{\circ} \mathrm{C}$ for one night. HRP-labeled secondary antibodies (goat anti mouse, 1:500) were added and incubated for $1 \mathrm{~h}$. Sections were sealed with neutral resin and pictures were taken using a microscope.

\section{Statistical analysis.}

Values for all continuous variables are expressed as the mean \pm standard error (SE) of the mean. Data were analyzed with Origin 8.0 software (Origin Lab Corp. Northampton, MA, USA). Data were evaluated by two-sample $t$ test for continuous variables and Chi-square test for categorical variables. $P<0.05$ was considered as a statistically significant result.

\section{Results}

\section{Patient data and tumor characteristics}


Characteristics of 13 patients with gastric polyps and 58 patients with gastric carcinoma were compared and analyzed (Table 1.). A significant difference was observed with respect to sex of the patients with gastric polyps (male account for $38.46 \%$ ) or the patients with gastric carcinoma (male account for $68.97 \%)\left(X^{2}=4.26, P<0.05\right)$. The ages of the patients with polyps or carcinoma showed no significantly different $(t=1.76, P=0.083)$. A significant difference was observed in peripheral CTC counts between patients with gastric polyps and patients with gastric carcinoma $(t=2.29, P=0.024)$.

Tissue differentiation of gastric polyps were primarily classified as low-grade intraepithelial neoplasia (38.46\%) and non-neoplastic proliferation (46.16\%), whereas tissue differentiation of gastric carcinoma was mainly classified as poorly differentiated adenocarcinoma (79.31\%). Adenomatous polyp was the most common pathology in gastric polyps (53.84\%), whereas tubular adenocarcinoma was the most common pathology in patients with gastric carcinoma (36.21\%; Table 1).

\section{Comparison of CTC counts between gastric polyps and gastric carcinoma}

Based on previous reports $[10,13,15-17]$ and our observation shown in Table 1 (gastric polyps, $0.69 \pm$ 0.17/3.2 ml; gastric carcinoma, $3.05 \pm 0.48 / 3.2 \mathrm{ml}$ ), CTC counts were divided into two groups: $>1 / 3.2 \mathrm{ml}$ and $\leq 1 / 3.2 \mathrm{ml}$. A significant difference was observed between gastric polyps and gastric carcinoma $\left(X^{2}\right.$ $=9.67, P<0.005$ ) (Table 2.), with higher CTC counts observed in gastric carcinoma, and lower CTC counts $(\leq 1 / 3.2 \mathrm{ml})$ observed in gastric polyps (Table 2.). Although there was an overlapping range (Table 1.) in CTC counts between gastric polyps (0-2) and gastric carcinoma (0-16), the difference observed was significant.

Table 2

CTC counts in gastric polyps and carcinoma

\begin{tabular}{|lllll|}
\hline & CTC counts $(>1 / 3.2 \mathrm{ml})$ & CTC counts $(\leq 1 / 3.2 \mathrm{ml})$ & $X^{2}$ Value & $P$ Value \\
\hline Polyps & 2 & 11 & $X^{2}=9.67$ & $P<0.005$ \\
\hline Carcinoma & 39 & 19 & & \\
\hline CTC, Circulating tumor cell & & & \\
\hline
\end{tabular}

\section{CTC counts and tissue pathological types in gastric polyps and gastric carcinoma}

When CTC counts in gastric polyps were divided into two groups ( $>1 / 3.2 \mathrm{ml}$ and $\leq 1 / 3.2 \mathrm{ml}$ ), only $15.38 \%$ polyps patients located in $>1 / 3.2 \mathrm{ml}$ group, while $84.62 \%$ polyps belonged to $\leq 1 / 3.2 \mathrm{ml}$ group (Table 3 .). No proliferative polyps were observed in $>1 / 3.2 \mathrm{ml}$ group, only part of adenomatous polyps was observed in $>1 / 3.2 \mathrm{ml}$ group $(28.57 \%)$ (Table 3 .). 
Table 3

CTCs counts according to tissue types in gastric polyps

\begin{tabular}{|lcccc|}
\hline CTC counts & \multicolumn{2}{c|}{ Adenomatous polyps } & Proliferative polyps & Percentage (\%) \\
\hline$>1 / 3.2 \mathrm{ml}$ group & 2 & 0 & $15.38 \%$ \\
\hline$\leqq 1 / 3.2 \mathrm{ml}$ group & 5 & 6 & & $84.62 \%$ \\
\hline CTC, Circulating tumor cell & & & \\
\hline
\end{tabular}

However, for gastric carcinoma patients, no well differentiation cases were identified in this study, but significant difference was observed between moderately differentiation group and poorly differentiation group ( $t=2.43, P=0.018$, Fig. 1.). No significant difference was detected across the tissue pathological types ( $P>0.05$; Table 4.), as compared between the divided groups, namely $>1 / 3.2 \mathrm{ml} v \mathrm{vs} . \leq 1 / 3.2 \mathrm{ml}$.

Table 4

CTC counts according to tissue pathological types in gastric carcinoma

\begin{tabular}{|c|c|c|c|c|}
\hline Carcinoma & CTC counts $(>1 / 3.2 \mathrm{ml})$ & CTC counts $(\leq 1 / 3.2 \mathrm{ml})$ & $X^{2}$ Value & $P$ Value \\
\hline Papillary & 3 & 4 & $X^{2}=0.76$ & $P>0.05$ \\
\hline Tubular & 17 & 4 & $X^{2}=0.39$ & $* P>0.05$ \\
\hline Papillarytubular & 2 & 1 & $X^{2}=0.72$ & ${ }^{\#} P>0.05$ \\
\hline Mucous & 13 & 5 & $x^{2}=2.05$ & $\triangle P>0.05$ \\
\hline Signet-ring cell & 4 & 5 & $x^{2}=2.49$ & $P>0.05$ \\
\hline
\end{tabular}

\section{CTC counts and tissue invasive depth in gastric carcinoma}

In gastric carcinoma patients, CTC counts was higher in mucous membrane layer $(3.0 \pm 0.33 / 3.2 \mathrm{ml})$ and serous layer $(2.56 \pm 0.39 / 3.2 \mathrm{ml})$, lower CTC count was observed in muscular layer $(1.4 \pm 0.4 / 3.2 \mathrm{ml})$, significant difference was observed between mucous membrane layer and muscular layer $(t=2.89, P=$ 0.012 , Fig. 2.), whereas significant difference was observed between serous layer and muscular layer $(\mathrm{t}=$ $0.97, P=0.333)$. Also, no significant difference was observed between serous layer and mucous membrane layer $(\mathrm{t}=0.19, P=0.849)$. These results suggested that CTC counts were higher in early gastric carcinoma (mucous membrane layer invasive), and CTC were detached easily in early gastric carcinoma other than advanced gastric carcinoma.

\section{CTC counts and lymph node positive rate in gastric carcinoma}


In this study, according to the percentage of lymph node metastasis, positive lymph node levels were divided into four grades (Table 5.) in gastric carcinoma patients, the relationship between CTC counts and different levels positive lymph node metastasis was analyzed. Interestingly, with the increase of positive lymph node percentage, CTC counts were gradually decreased (Table 5.), but no significant difference was observed among different levels of positive lymph node metastasis ( $P>0.05$; Table 5.). This result indicated that CTC counts were higher in early gastric carcinoma (lower positive lymph node levels), and CTC were easily detached in early gastric carcinoma than that in advanced gastric carcinoma.

Table 5

CTCs counts according tolymph node positive rate in gastric carcinoma

\begin{tabular}{|lllll|}
\hline Lymph node (positive/total) & CTCs count $(\mathrm{n} / 3.2 \mathrm{ml})$ & $\mathbf{N}$ & $t$ Value & $P$ Value \\
\hline$\leq 10 \%(0-4) /(10-49)$ & $3.50 \pm 0.96$ & 22 & $t=0.06$ & $P>0.05$ \\
\hline$>10 \%, \leq 30 \%(1-6) /(5-36)$ & $3.60 \pm 1.56$ & 10 & $t=0.70$ & $* P>0.05$ \\
\hline$>30 \%, \leq 60 \%(6-26) /(13-44)$ & $2.42 \pm 0.83$ & 12 & $t=0.15$ & ${ }^{*} P>0.05$ \\
\hline$>60 \%, \leq 100 \%(5-26) /(7-41)$ & $2.25 \pm 0.41$ & 8 & $t=0.75$ & $\Delta P>0.05$ \\
\hline $\begin{array}{l}\text { Values are expressed as the mean } \pm \text { standard error of the mean. CTC, Circulating tumor cell. } P=(\leq \\
10 \%) v s(>10 \%, \leq 30 \%), * P=(>10 \%, \leq 30 \%) \text { vs }(>30 \%, \leq 60 \%),{ }^{*} P=(>30 \%, \leq 60 \%) \\
(>60 \%, \leq 100 \%) \text { vs }(>10 \%, \leq 30 \%) .\end{array}$ & & & \\
\hline
\end{tabular}

\section{Comparison of positive rate of CTC counts and tumor markers in gastric carcinoma}

Tumor markers CEA, CA19-9, CA72-4 were detected in non-metastatic gastric cancer in this study, only $13.79 \%$ positive rate was shown in gastric carcinoma patients when CEA acted as an index to reflect the existence of gastric carcinoma, and $12.07 \%$ positive rate with CA19-9, 17.24\% positive rate with CA72-4, respectively (Table 6.). Even the joint detection of CEA, CA19-9 and CA72-4, the positive rate was reached at merely $29.31 \%$ (Table 6.), which was far below the positive rate of CTCs > 1/3.2 $\mathrm{ml}$ criterion $(67.24 \%)$ and CTC $0-1 / 3.2 \mathrm{ml}$ criterion (79.31\%). CTC criterion possesses higher positive rate than CEA, CA19-9 or CA72-4 when they acted as an sole index to reflect the existence of gastric carcinoma, significant differences in positive rate were observed between CTC $>1 / 3.2 \mathrm{ml}$ criterion and CEA, CA19-9 and CA72-4 $\left(X^{2}=34.37, X^{2}=36.89, X^{2}=29.72\right.$, respectively, $P<0.001$, Table 6.). There is significant difference, when compared the positive rate of the joint detection of CEA, CA19-9 and CA72-4, with either results from CTC $>1 / 3.2 \mathrm{ml}$ criterion or from CTC $0-1 / 3.2 \mathrm{ml}$ criterion $\left(X^{2}=29.22\right.$ (vs. CTC > 1/3.2 ml), $X^{2}=16.71$ (vs. CTC 0-1/3.2 ml), ${ }^{\#} P<0.001$,Table 6.). 
Table 6

Comparison of positive rate of CTC countsand tumor markers in gastric carcinoma

\begin{tabular}{|c|c|c|c|c|}
\hline Variable & Positive & Negative & $X^{2}$ Value & P Value \\
\hline CEA & $8(13.79 \%)$ & $50(86.21 \%)$ & $x^{2}=34.37$ & ${ }^{\#} P<0.001$ \\
\hline CA19-9 & $7(12.07 \%)$ & $51(87.93 \%)$ & $x^{2}=36.89$ & ${ }^{\#} P<0.001$ \\
\hline CA72-4 & $10(17.24 \%)$ & $48(82.76 \%)$ & $X^{2}=29.72$ & ${ }^{\#} P<0.001$ \\
\hline${ }^{\triangle} C E A+C A 19-9+C A 72-4$ & $17(29.31 \%)$ & $41(70.69 \%)$ & & \\
\hline \multicolumn{5}{|l|}{ CTCs count (n/3.2 ml) } \\
\hline$n=0-1$ & $46(79.31 \%)$ & $12(20.69 \%)$ & $x^{2}=29.22$ & $\Delta P<0.001$ \\
\hline$\# n>1$ & $39(67.24 \%)$ & $19(32.76 \%)$ & $x^{2}=16.71$ & $\Delta P<0.001$ \\
\hline \multicolumn{5}{|c|}{ 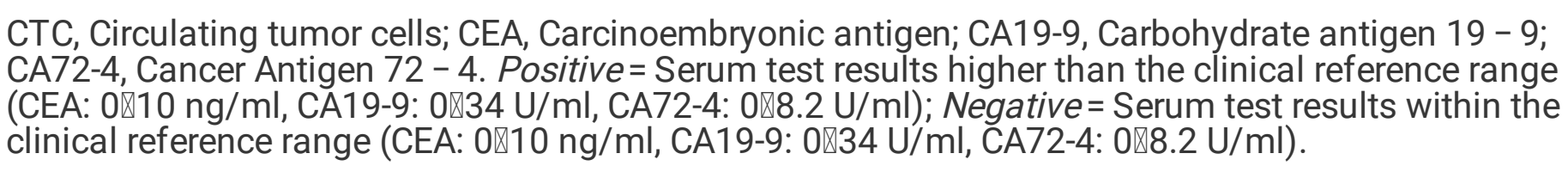 } \\
\hline
\end{tabular}

\section{Discussion}

The detection of CTCs has been widely reported for the diagnostic and predictive properties in numerous types of cancers, such as breast cancer, prostate cancer, colorectal cancer, gastric cancer [5-7]. Clinical implications of CTCs, including screening tool for early diagnosis, biomarkers to specific therapies, monitoring tool to assess the response of chemotherapies. Research focusing on the association of CTCs and postoperative recurrence, as well as response to chemotherapies has been extensively conducted, whereas the application of CTCs in the early diagnosis received limited attention. Moreover, the use of this system for screening, mainly compared the malignant tumor with the healthy control [17] due to the sensitivity and specificity of detection technique. Nevertheless, the differentiation between cancer and benign diseases (e.g. Polyp and cancer) determined by CTCs has been rarely reported, among which the clinical implication of CTCs was associated to clinical symptomatic parameters, such as the tumor stage, histopathological type, degree of differentiation, depth of invasion, and lymph node metastasis. In the current study, aside from the conventional comparison between cancer patients and their healthy controls, the association between the existence of polyps and CTCs was also analyzed, based on tumor biomarkers, which could provide information for the diagnosis of tumor in early and intermediate stage.

CTCs are extensively distributed in the blood of all types of cancer patients, as the development of the research, the CTCs has been detected almost everywhere, even in the blood sample of tumor free individuals and patients bearing benign tumor $[11-13,17]$. If the peripheral CTCs was also detectable in patients with gastric polyps, how about that in those representative benign tumor-like lesions? If it is 
possible, would the CTC counting interval be different, comparing to healthy controls and malignant patients. The current study was designed to determine those questions by the observation of CTCs between patients with gastric polyps and malignancy tumor. Our results showed that the CTC counting can be identified in peripheral circulation of patients with either polyps or gastric carcinoma, however, the polyps group showed a lower CTCs $(0.69 \pm 0.17 / 3.2 \mathrm{ml})$, comparing to that of their counterparts $(3.05 \pm$ $0.48 / 3.2 \mathrm{ml}$ ). Although there is a certain overlap of the two intervals, significant difference was found among the two groups $(P<0.05)$. The results suggested that the higher CTC counting is associated with higher risk of cancer, therefore CTCs could be adopted as a valuable cancer screening biomarker.

According to the previous publications and our experience, the CTCs counting number were divided into two groups, $>1 / 3.2 \mathrm{ml}$ and $\leq 1 / 3.2 \mathrm{ml}[10,13,15-17]$. Alvarez Cubero[18] reported that the number of CTCs from metastatic tumor is $1-10 / \mathrm{ml}$, while that of non-metastatic tumor is $0.5-5 / \mathrm{ml}$, whose lowest value $(0.5 / \mathrm{ml})$ is close to our criteria $(1 / 3.2 \mathrm{ml})$. Our study found that the probability of malignant tumor in CTC value over $(1 / 3.2 \mathrm{ml})$ is $67.24 \%$, meanwhile, the rate for benign tumor in CTC value below $(1 / 3.2 \mathrm{ml})$ is $84.62 \%$. Additionally, we found that there is a significant difference between patients with gastric polyps and gastric carcinoma $\left(X^{2}=9.67, \mathrm{P}<0.005\right)$. The highest CTC counting is mostly detected in carcinoma patients, indicated that CTCs could be used for the preliminary clarify benign and malignant.

For the early stage resectable gastroenteric cancer, the tumor specimens can be collected to determine the differentiation, invasion depth including peripheral vessel, lymphangion and nerves, as well as the lymphatic metastasis. As for patients with gastric polyps, the pathological type can be identified by biopsies after endoscopic polypectomy. In the current study, a refined clinical correlation analysis was carried out by the clinical pathological stage combined with the CTCs, provided reference for the application of CTCs monitor for early stage.After comparing the gastric carcinoma pathological type of two CTCs groups ( $>1 / 3.2 \mathrm{ml}$ vs. $\leq 1 / 3.2 \mathrm{ml})$, there is no significant difference of the distribution of pathological types among two groups $(P>0.05)$, suggested that CTCs is not influenced by the pathological types. The gradient analysis based the positive rate of lymphatic metastasis, showed that the higher CTCs group showed a relative low rate of lymphatic metastasis, is negatively correlated, though needs further determination. As for the depth of tumor invasion, lower CTC counting was observed in muscular layer, while higher numbers in mucous membrane layer and serous layer. Significant difference was observed in muscular layer, compared with the other two layers $(P<0.05)$, indicating that the higher CTCs is possible detected in the early gastric carcinoma, which therefore could be used as an indicator for early gastric carcinoma roughly screening.

CEA, CA19-9 and CA72-4 have long been employed for tumor preliminary screening, especially CEA in monitoring the gastroenteric tumor relapse. There is no improved tumor marker has been identified, although these biomarkers possess limited value in tumor early screening. Our results suggested that the CTCs counting showed superior value in early monitor comparing to CEA, CA19-9 and CA72-4, which could be developed as one of the most powerful approaches for early gastroenteric tumor regardless of the higher expense [17]. 


\section{Conclusion}

CTCs exist widely in patients either with gastric polyps or gastric carcinoma, however the latter showed a dramatic higher counting number. CTCs could be used as a predictor for the existence of gastroenteric neoplastic disorders, which is helpful to roughly tell the benign and malignant of tumor, furtherly, it showed a better reflection on early tumor development comparing to the conventional tumor marker, such as CEA, CA19-9 and CA72-4.

\section{Declarations}

\section{Ethics approval and consent to participate}

The study was carried on after acquiring patients' consent and Tongren Hospital ethics committee approval and consent.

\section{Consent for publication}

Not applicable.

\section{Availability of data and material}

Data sharing not applicable to this article as no datasets were generated or analyzed during the current study.

\section{Competing interests}

We declare that we have no competing interests.

\section{Authors' contributions}

We believe this work is scientifically valid, and all authors have sufficiently contributed to the study. Jun $\mathrm{He}$, Yingjie Xu, Peng Sun conceived of the study, and participated in its design and helped to draft the manuscript, Chen Qian, Jun Yan participated in its design and data collection, Chengguang Yang conceived of the study and draft the manuscript. 


\section{Funding}

The research fundingon CTCs detection was obtained from patients' medical insurance supported

by the national health care costs. This work supported by the key medical specialty of Shanghai (Grant No. ZK2019A15).

\section{Acknowledgments}

Not applicable.

\section{References}

1. Dominguez RL, Cherry CB, Estevez-Ordonez D, Mera R, Escamilla V, Pawlita M, Waterboer T, Wilson KT, Peek RM, Tavera G, Williams SM, Gulley ML, Emch M, Morgan DR. Geospatial analyses identify regional hot spots of diffuse gastric cancer in rural Central America. Med J Aust.BMC Cancer. 2019 Jun 7;19(1):545.

2. Kim GH, Liang PS, Bang SJ, Hwang JH. Screening and surveillance for gastric cancer in the United States: Is it needed? Gastrointest Endosc. 2016 Jul;84(1):18-28.

3. Suh YS, Yang HK. Screening and Early Detection of Gastric Cancer: East Versus West.Surg Clin North Am. 2015 Oct;95(5):1053-66.

4. Mitani S, Kadowaki S, Hasegawa H, Wakatsuki T, Hara H, Tajika M, Nishikawa K, Hirao M, Takahari D, Chin K, Muro K. Systemic chemotherapy for gastric cancer with early recurrence after adjuvant S-1 monotherapy: a multicenter retrospective study. Int J Clin Oncol. 2019 May 31.

5. Cohen SJ, Punt CJ, lannotti N, et al. Relationship of circulating tumor cells to tumor response, progression-freesurvival, and overall survival in patients with metastatic colorectal cancer. J Clin Oncol. 2008;26(19):3213-21.

6. de Bono JS, Scher HI, Montgomery RB. et.al. Circulating tumor cells predict survival benefit from treatment in metastatic castration-resistant prostate cancer. Clin Cancer Res. 2008;14(19):6302-9.

7. Cristofanilli M, Budd GT, Ellis MJ, et al. Circulating tumor cells,disease progression, and survival inmetastatic breast cancer. N Engl JMed. 2004;351(8):781-91.

8. Bork U, Rahbari NN, Schölch S, Reissfelder C, Kahlert C, Büchler MW, Weitz J, Koch M. Circulating tumour cells and outcome in non-metastatic colorectal cancer: a prospective study. $\mathrm{Br} \mathrm{J}$ Cancer. 2015 Apr;14(8):1306-13. 112.

9. Zheng L, Zou K, Yang C, Chen F, Guo T, Xiong B. Inflammation-based indexes and clinic- pathologic features are strong predictive values of preoperative circulating tumor cell detection in gastric cancer patients.Clin Transl Oncol. 2017 Sep;19(9):1125-1132. 
10. Han L, Li YJ, Zhang WD, Song PP, Li H, Li S. Clinical significance of tumor cells in the peripheral blood of patients with esophageal squamous cell carcinoma.Medicine (Baltimore). 2019 Feb;98(6):e13921.

11. Pantel $K$, Denève $E$, Nocca $D$, Coffy $A$, Vendrell JP, Maudelonde T, Riethdorf $S$, Alix-Panabières $C$. Circulating epithelial cells in patients with benign colon diseases. Clin Chem. 2012;58:936-40.

12. Tsai W-S, Chen J-S, Shao H-J, Wu J-C, Lai Jr-Ming, Lu S-H, Hung T-F, Chiu Y-C, You J-F, Hsieh P-S, Yeh $\mathrm{C}-\mathrm{Y}$, Hung H-Y, Chiang S-F, Lin G-P, Tang R-P, Chang Y-C. Circulating tumor cell count correlates with colorectal neoplasm progression and is a prognostic marker for distant metastasis in non-metastatic patients. Sci Rep. 2016;6:24517.

13. Yang C, Zhuang W, Hu Y, Zhu L. Clinical significance of peripheral circulating tumor cell counts in colorectal polyps and non-metastatic colorectal cancer. World J Surg Oncol. 2018 Jan 22;16(1):13.

14. Ahsen O. Circulating Gorges TM, Tinhofer I, Drosch M, Röse L, Zollner TM, Krahn T von. Ahsen O. Circulating.

15. tumour cells escape. from EpCAM-based detection due to epithelial-to-mesenchymal transition. BMC Cancer. 2012 May;16:12:178.

16. Thierry $B$ Detectionand clinical Hardingham JE, Grover P, Winter M, Hewett PJ, Price TJ. Thierry B. Detectionand clinical.

17. significance of circulating. tumor cells in colorectal cancer-20 years of progress. Mol Med.2015 Oct 27;21 Suppl 1:S25-31.

18. Cohen SJ, Punt CJ, lannotti N, Saidman BH, Sabbath KD, Gabrail NY, Picus J, Morse M, Mitchell E, Miller MC, Doyle GV, Tissing H, Terstappen LW, Meropol NJ. Relationship of circulatingtumor cells to tumor response, progression-freesurvival, and overall survival in patients withmetastatic colorectal cancer. J Clin Oncol. 2008 Jul;1:26:3213-21.

19. Kang HM, Kim GH, Jeon HK, Kim DH, Jeon TY, Park DY, Jeong H, Chun WJ, Kim MH, Park J, LimM, Kim TH, Cho YK. Circulating tumor cells detected by lab-on-a-disc: Role in early diagnosis of gastric cancer. PLoS ONE. 2017;12(6):e0180251.

20. Alvarez Cubero MJ, Lorente JA, Robles-Fernandez I, Rodriguez-Martinez A, Puche JL, Serrano MJ. Circulating Tumor Cells: Markers and Methodologies for Enrichment and Detection. Methods Mol Biol. 2017;1634:283-303.

\section{Figures}




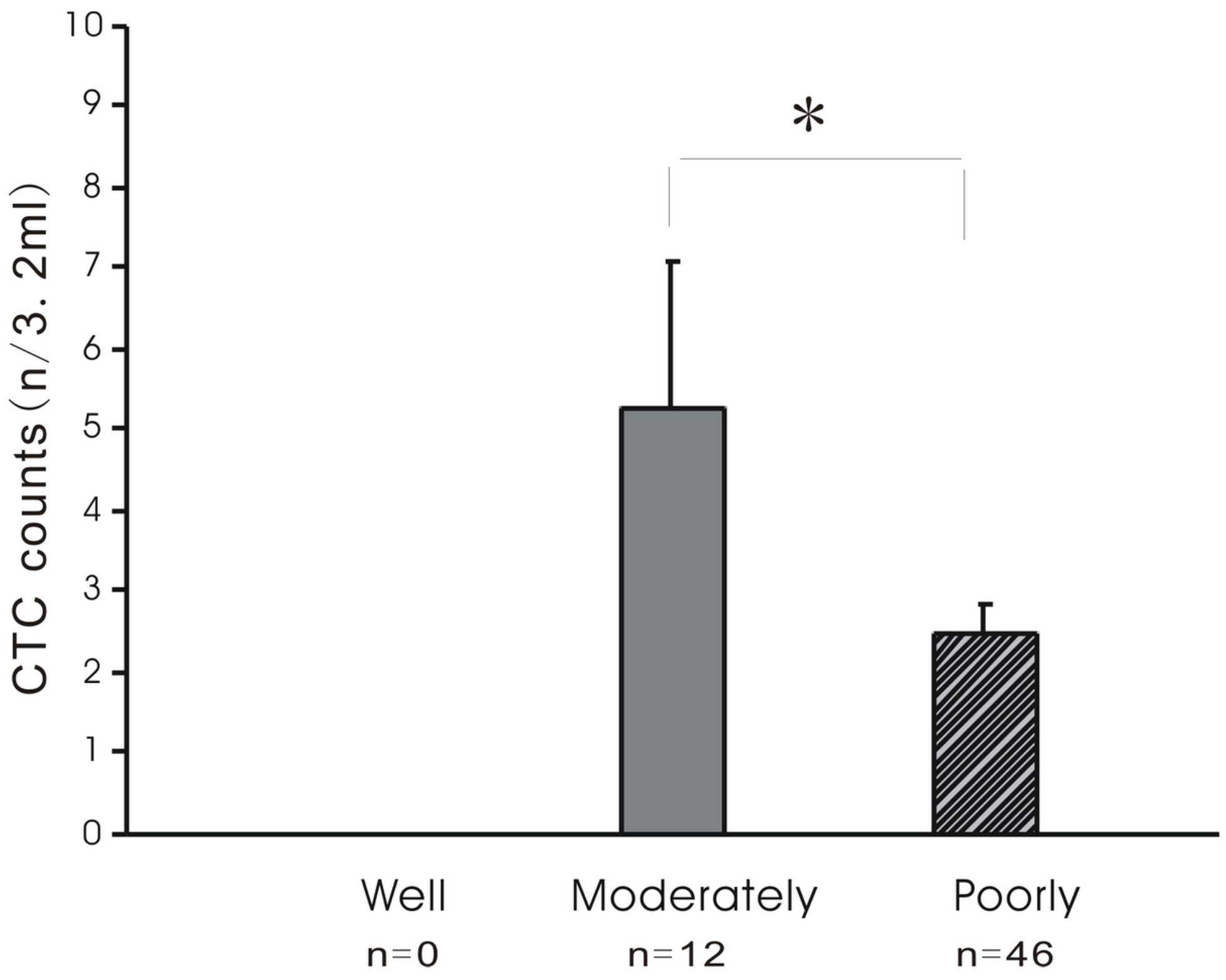

Figure 1

CTC counts in different tissue differentiations in gastric carcinoma, moderately differentiated group versus poorly differentiated group $\left(t=2.43,{ }^{\star} P \otimes 0.05\right)$. 


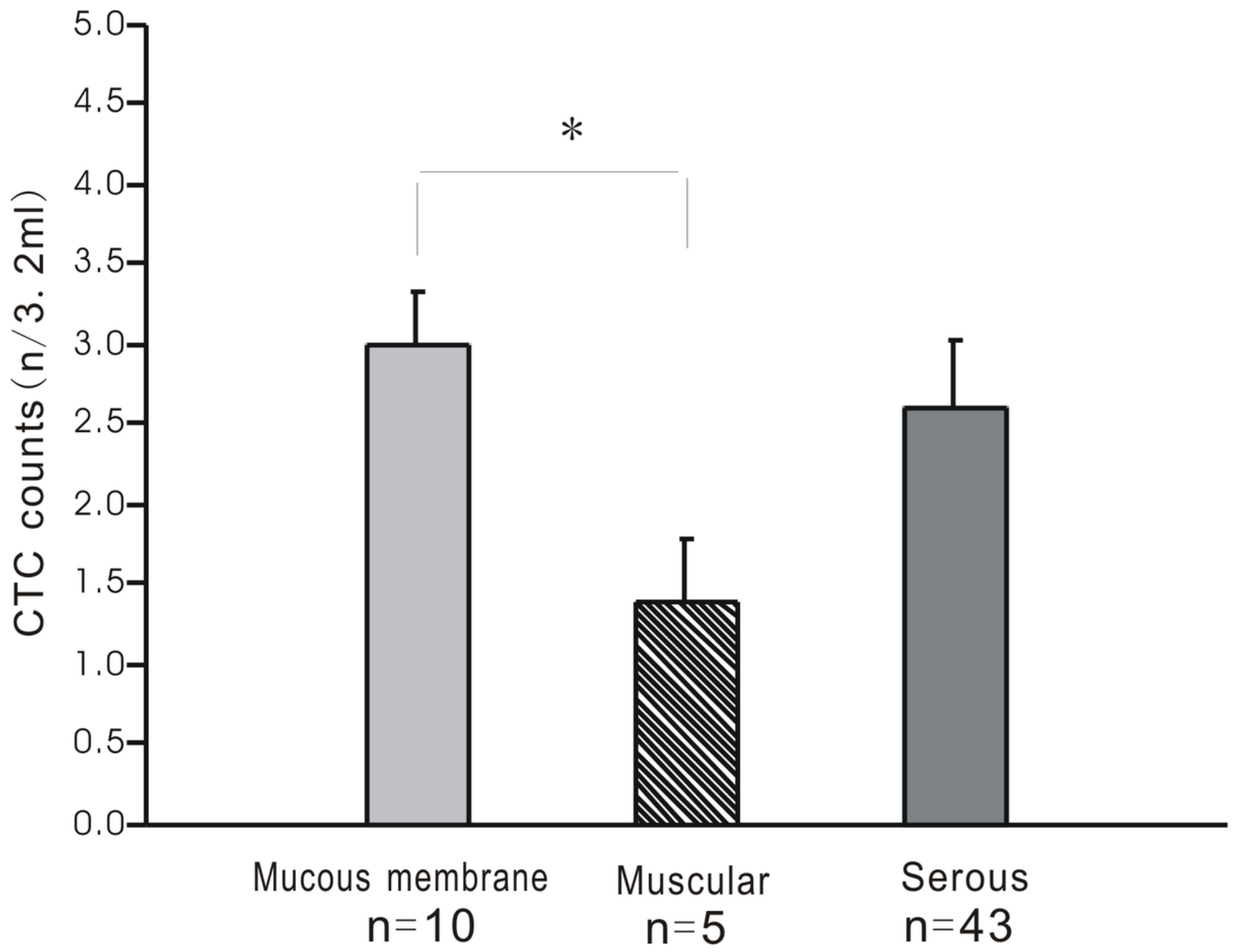

Figure 2

CTC counts in different tissue invasive depth in gastric carcinoma, mucous membrane layer versus muscular layer $(t=2.89, * P \otimes 0.05)$; serous layer versus muscular layer $(t=0.97, P \otimes 0.05)$. 